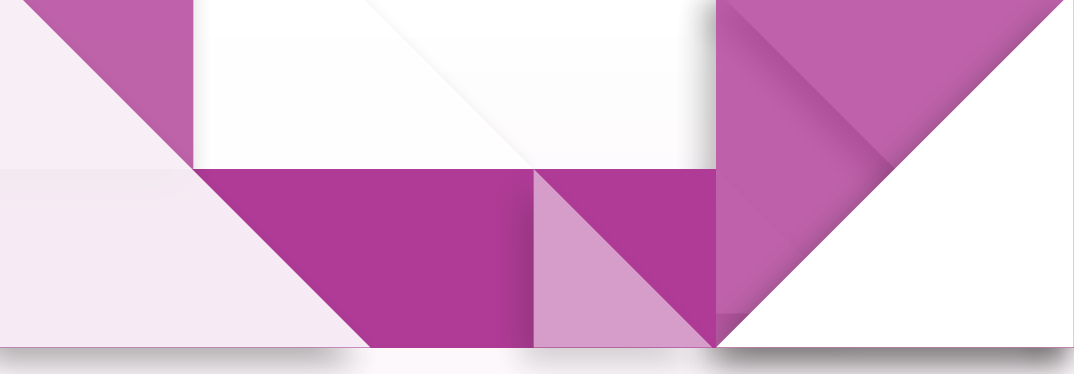

\title{
1) La actividad experimental 3 como una parte fundamental para la enseñanza de la física moderna: el caso de la mecánica cuántica
}

\section{- Experimental Activity as a Fundamental Part for Teaching of Modern Physics: The Case of Quantum Mechanics \\ - Atividade experimental como parte fundamental do ensino da física moderna: o caso da mecânica quântica}

\section{Resumen}

En las últimas décadas se han propuesto metodologías y estrategias para la enseñanza de la mecánica cuántica. Esto se debe a que ha generado una revolución cognitiva en la medida que da una nueva visión del mundo. Esta es una de las razones por la que es importante enseñarla y a la vez permite profundizar en las dificultades que presentan los estudiantes en la apropiación de algunos conceptos en los que se fundamenta como son: la noción de estado y principio de superposición. En este sentido, se propone un enfoque fenoménico como herramienta para la enseñanza de la mecánica cuántica. Bajo este contexto, se desarrollan actividades experimentales que son la estrategia para involucrar a los estudiantes en la construcción de explicaciones alrededor del fenómeno. La actividad experimental es entendida como aquella que le permite al estudiante realizar una reflexión alrededor del fenómeno para su explicación. Este enfoque favorece el desarrollo de un pensamiento físico ya que el estudiante involucra habilidades y maneras de proceder, maneras de pensar y maneras de construir y reconstruir argumentos para la explicación del fenómeno considerado. Esta forma de plantear la enseñanza de la mecánica cuántica supera la dicotomía entre experimento y teoría ya que desde esta perspectiva el experimento es considerado parte constituyente para la construcción de explicaciones en torno al fenómeno.

Palabras clave

mecánica cuántica; enseñanza; actividad experimental; enfoque fenoménico-conceptual.

\section{Mauricio Rozo Clavijo* Angélica Walteros* Carlos Cortés ${ }^{* * *}$}

* Maestría en Ciencias Físicas, Universidad
Pedagógica Nacional, investigador principal,
https://orcid.org/0000-0001-6427-8608.
mclavijo@pedagogica.edu.co
** Licenciada en Física, Liceo Parroquial San
José, coinvestigadora, https://orcid.org/0000-
0001-9006-9535.
angelicawalteros94@gmail.com

*** Licenciado en Física, Colegio Parroquial Confraternidad de la Doctrina Cristiana, coinvestigador, https://orcid.org/0000-0002-97201305. Universidad Pedagógica Nacional. dfi_cgcortesh288@pedagogica.edu.co

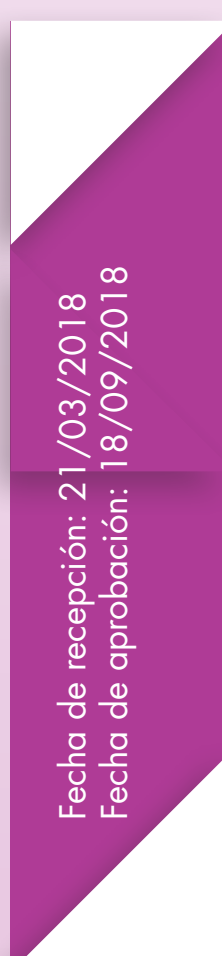




\begin{abstract}
Over the last few decades, methodologies and strategies have been proposed for the teaching of quantum mechanics. This is because it has generated a cognitive revolution insofar as it offers a new view of the world. This is one of the reasons why it is important to teach it and at the same time it allows to delve deeper into the difficulties that the students face when appropriating the concepts on which it is based as they are: the notion of state and the principle of superposition. In this sense, a phenomenological approach is proposed as a tool for teaching quantum mechanics. In this context, experimental activities are developed, which are the strategy to involve the students in building explanations about the phenomenon. The experimental activity is understood as that which allows the student to reflect on the phenomenon in order to explain it. This approach favors the development of a physical thought since the student involves skills and ways of proceeding, ways of thinking and ways of constructing and reconstructing arguments to explain the phenomenon under consideration. This way of proposing the teaching of quantum mechanics overcomes the dichotomy between experiment and theory since from this approach the experiment is considered as a constituent part for the construction of explanations about the phenomenon.
\end{abstract}

Keyword

Quantum mechanics; teaching; experimental activity; phenomenological-conceptual

\title{
Resumo
}

Nas últimas décadas tem-se proposto metodologias e estratégias para o ensino da mecânica quântica. Deve-se isto a que se tem gerado uma revolução cognitiva na medida que se dá uma nova visão do mundo. Esta é uma das razões pelas quais é importante ensina-la e por sua vez permite aprofundar nas dificuldades que apresentam os estudantes na apropriação dos conceitos nos quais fundamenta-se como os são: a noção de estado e princípio de supercompensação. Nesse sentido, se propõe um enfoque fenomênico como ferramenta para o ensino da mecânica quântica. Assim nesse contexto, se desenvolvem atividades experimentais que são a estratégia para involucrar aos estudantes na construção de explicações ao redor do fenômeno. A atividade experimental é entendida como aquela que permite ao estudante refletir sobre o fenômeno para sua explicação. Este enfoque favorece desenvolver de um pensamento físico já que o estudante involucra habilidades e formas de proceder, maneiras de pensar e formas de construir e reconstruir argumentos para a explicação do fenômeno considerado. Esta forma de propor o ensino da mecânica quântica supera a dicotomia entre experimento e teoria logo que desde este enfoque o experimento é considerado como parte constituem-te para a construção de explicações em torno ao fenômeno. Finamente, espera-se que os estudantes adquiram um conhecimento com sentido e significado baixo a perspectiva proposta.

Palavras-chave

mecânica quântica; ensino; atividade experimental; enfoque fenomênico-conceitual 


\section{Introducción}

La indagación sobre qué es la materia condujo a los pensadores del siglo XIX a la elaboración de experimentos cuyos resultados pusieron en evidencia que tanto la mecánica newtoniana como la teoría clásica de campos, hasta entonces formuladas, no daban respuesta satisfactoria a algunos de los datos empíricos obtenidos. Es así que estas teorías no dan respuesta a preguntas como: ¿̇la materia tiene estructura?, y si la tiene ¿̇cómo se pueden explicar las propiedades de los elementos a partir de ésta?

La teoría cuántica surge como respuesta a estas preguntas y muestra además una visión de mundo caracterizada por el hecho de que la observación que se realiza sobre el sistema tiene un grado incontrolable de perturbación sobre este, es decir, el observador afecta y define la realidad que estudia (Dirac, 1967). La visión que demanda la mecánica cuántica no solo es extraña sino que es más extraña de lo que se puede suponer. No tiene sentido que un objeto pueda estar en dos sitios a la vez. Nuestro sentido común o intuición nos dice que esto no puede ocurrir y va en contravía con la manera de conocer que tiene el sujeto en su interacción con la naturaleza.

La mecánica cuántica pone en aprietos nuestra intuición al establecer que la propia observación crea la realidad observada. Desde este punto de vista, resulta difícil para el estudiante formarse una imagen mental (Mengual, 2005) sobre el objeto de estudio sin cometer grandes errores como lo sugiere Dirac, "[...] las leyes fundamentales no rigen el mundo directamente tal como este aparece en nuestra imagen mental, sino que actúa sobre un sustrato del que no podemos formarnos ninguna imagen mental sin cometer desatinos" (Dirac, 1967).
Por otra parte, el esquema que brinda la física clásica condiciona la manera de ver el mundo dado que, en general, toda explicación que se realiza de un fenómeno, independientemente de su naturaleza, se hace a partir de modelos construidos con elaboraciones que están a la base de los fenómenos mecánicos. Este esquema establece que cualquier fenómeno es comprendido si es explicado en términos mecánicos (Schmid, 1984). Bajo este contexto, en general, se instaura a la mecánica, de corte newtoniano, como la puerta de entrada a la física y a su enseñanza, lo cual se sustenta con el estudio de esta en los primeros años de la vida escolar de los estudiantes, determinando la comprensión e ideas de estos, además de no permitirles pensar de manera diferente al esquema clásico (Joshua et al., 2005).

Esta es la manera en que se plantea usualmente la enseñanza de la física y que hoy se sigue proponiendo sin tener presente la cosmovisión de mundo que nos ofrece la mecánica cuántica. La física del siglo xx ha modificado nuestra imagen newtoniana del mundo de manera radical, además ha permitido un avance tecnológico para beneficio - bienestar del mismo hombre. La nueva comprensión del mundo se fundamenta en dos grandes teorías: la relatividad general y la mecánica cuántica. Ambas teorías exigen una modificación radical sobre nuestra manera de conocer y sobre las ideas convencionales que se tienen acerca del mundo, poniendo de manifiesto un revolucionario alcance conceptual.

El rápido avance de la tecnología torna imperioso proponer estrategias de enseñanza que permitan abordar temas alrededor de la mecánica cuántica en los cursos introductorios de física en la universidad, con el fin de influir en la formación inicial de los profesores y a la vez romper con el paradigma newtoniano que 
ha imperado e influido en nuestro pensamiento y la manera de ver el mundo desde el siglo XVIII hasta nuestros días.

En la última década se han propuesto metodologías y estrategias para la enseñanza de la mecánica cuántica (Beltrán y Mosquera, 2016; Moreira et al., 2000). Esto se debe a que ha generado una revolución cognitiva en la medida que da una nueva visión del mundo. Esta es una de las razones por las que es importante enseñarla y a la vez permite profundizar en las dificultades que presentan los estudiantes en la apropiación de los conceptos en los que se fundamenta, y además, permite plantear una serie de interrogantes a los docentes como: ¿̇qué papel desempeñan la formalización y la actividad experimental para la comprensión de los fenómenos atómicos? ¿ Cómo es posible promover un pensamiento científico en los estudiantes desde la física que se enseña?

Una de las mayores dificultades para la enseñanza de la mecánica cuántica se debe a que los fenómenos cuánticos no son directamente perceptibles y, por lo tanto, son contrarios a la intuición que deriva de la percepción de los estudiantes, y más aún cuando el docente los aborda desde una perspectiva teórica en la que desarrolla el formalismo sin resaltar las implicaciones que conllevan una visión cuántica del mundo (Ferreyra y González, 2000; Gill, 1983).

Por lo tanto, se propone un enfoque fenoménico como herramienta para la enseñanza de la mecánica cuántica. Bajo este contexto, se plantean y desarrollan actividades experimentales entendidas como la estrategia para involucrar a estudiantes y docentes en la construcción de explicaciones alrededor del fenómeno. Este enfoque favorece el desarrollo de un pensamiento físico ya que el estudiante involucra habilidades y maneras de proceder, maneras de pensar y maneras de construir y reconstruir argumentos para la explicación del fenómeno. Esta forma de plantear la enseñanza de la mecánica cuántica supera la dicotomía entre experimento y teoría ya que desde esta perspectiva el experimento es considerado parte de la construcción de explicaciones en torno al fenómeno.

Se clarifica, además, que las actividades experimentales propuestas son de óptica clásica, de manera que los conceptos que inicialmente construyen los estudiantes son propios de la física clásica, no obstante, en las actividades se propende por el abandono del proceder clásico de conocer, lo cual da la oportunidad de inaugurar una nueva forma de conocer fundamentada en una elaboración apoyada en un conocer cuántico, por lo tanto, la explicación del fenómeno exige del abandono del proceder clásico de conocer lo cual constituye una primera aurora sobre un pensamiento cuántico.

Bajo este contexto, se propende estimular en los estudiantes un conocer cuántico y confrontarlos con tres aspectos claves de la mecánica cuántica: 


\section{El carácter corpuscular de la materia y la radiación}

La manera clásica de conocer plantea un soporte material para la radiación denominado éter debido a su carácter ondulatorio. Sin embargo, la radiación, teniendo un comportamiento corpuscular, puede ser considerada un campo cuántico, enjambres de corpúsculos siendo de evidencia experimental el carácter discreto de la energía. Es así que la energía del campo electromagnético puede tomar ciertos valores determinados $y$, por lo tanto, revela un comportamiento corpuscular. De igual manera, la materia se muestra con un comportamiento corpuscular, no obstante, también manifiesta un comportamiento ondulatorio evidenciado en los fenómenos de difracción, lo cual constituye una nueva manera de conocer respecto a esta. No solo los campos interactúan como partículas sino también las partículas se reparten por el espacio-tiempo como si fueran campos. La noción de campo y partícula se unifican desde el contexto de la mecánica cuántica. El carácter granular de la materia y de la radiación son características propias de los sistemas cuánticos, a diferencia del pensamiento clásico que plantea una energía con un carácter continuo para la descripción los sistemas objeto de estudio.

\section{El indeterminismo}

El mundo físico es la sucesión de eventos cuánticos posibles en el espacio-tiempo. Estos eventos se caracterizan por la interacción del sistema cuántico con otros. Dado que al sistema cuántico no se le atribuye trayectoria, aparece y desaparece en un determinado lugar sin preverlo con certeza, el futuro es imprevisible; futuro que no está determinado unívocamente por un pasado, es decir, el sistema cuántico tiene muchas posibilidades de estar, contrario al determinismo de causa-efecto dado para un sistema desde el contexto clásico.

\section{La observación y el relacionismo}

El observador perturba de manera incontrolable al sistema cuántico y crea la realidad que observa, es decir, las cosas son reales en relación con la interacción asociada; antes de la observación no se tiene conocimiento real del sistema cuántico. La realidad no es más que la interacción del sistema cuántico con el sistema clásico, no se puede describir la realidad esencial de un sistema que no interactúa. Por lo tanto, la realidad de una cosa depende de la interacción con las otras cosas.

El mundo físico desde el contexto de la mecánica cuántica es un mundo de eventos en donde los sistemas cuánticos adquieren realidad en el momento de la observación, es decir, cuando existe una interacción con el sistema macroscópico. En este sentido, la mecánica cuántica describe procesos y eventos de sistemas que interaccionan con otros, y no describe en sí mismo al sistema. A diferencia del observador clásico que no perturba de manera apreciable al sistema cuando hace una medición sobre este y, por lo tanto, no crea la realidad que observa, es decir, la realidad clásica es independiente de la interacción.

Las limitaciones de nuestra intuición nos muestran la mecánica cuántica como ininteligible. Por lo tanto, se propende estimular en los estudiantes una "intuición cuántica" a partir de un conocer cuántico haciendo uso de actividades experimentales como: el espectro de la luz visible y el experimento de la polarización de la luz, con los cuales se espera que los estudiantes puedan superar las ideas clásicas y les permita clarificar los conceptos de estado, principio de superposición, amplitud de probabilidad y probabilidad.

Esta manera de plantear la enseñanza de la mecánica cuántica da la posibilidad de examinar el contexto problemático al que 
responde, los fundamentos, las concepciones y los modos de razonamiento implicados en la teoría y provee un espacio para realizar una exploración sobre las nociones, esquemas y experimentos que los pensadores emplearon para la construcción y fundamento de esta.

La propuesta tiene como problema central la enseñanza de la física moderna, el caso de la mecánica cuántica en los niveles introductorios de educación universitaria haciendo uso de la metodología fenoménica. La enseñanza de la mecánica cuántica entendida bajo esta perspectiva, no privilegia los conceptos y teorías formuladas por los científicos sino que las pone al mismo nivel con las elaboraciones que realizan los profesores y estudiantes (Moreira et al., 2004; Otero et al., 2009). La propuesta está dirigida a profesores y estudiantes de secundaria, así como a profesores en formación a nivel universitario con el fin de lograr un cambio de paradigma en la enseñanza de la física. Además, se busca aportar elementos que permitan una transformación en las prácticas de enseñanza de la física y en particular de la mecánica cuántica (Hodson, 1994).

Finalmente, esta perspectiva permite desarrollar en el campo de la enseñanza de la física y, en particular, de la mecánica cuántica, una formación investigativa además de una cultura científica contemporánea con sentido y significado, superando las dificultades y obstáculos que presentan los estudiantes en su aprendizaje, contribuyendo en una formación de docentes en física y buscando un cambio de paradigma, un cambio en las prácticas de enseñanza y en la visión del mundo.

\section{El papel del experimento para la enseñanza de la mecánica cuántica}

En la última década se han realizado una serie de reflexiones (Solbes et al., 2010) sobre las dificultades en relación con la enseñanza-aprendizaje de la mecánica cuántica (Solbes et al., 1988). En estas se evidencian una serie de papeles en los que se muestra al docente, en la mayoría de los casos, a la simple descripción de los fenómenos por medio de experimentos y ecuaciones sin resaltar la manera como los conceptos y los procesos de formalización surgen dentro de un contexto particular (Fanaro, 2009; Greca et al., 2002). Además, los temas abordados, en la mayoría de los casos, se muestran bajo un esquema cronológico sobre el desarrollo histórico de la mecánica cuántica con la finalidad de persuadir a los estudiantes sobre las diferentes concepciones a las que se llegaron a través de razonamientos como consecuencia de experimentos cruciales.

Considerando las dificultades y la forma como se plantea la enseñanza de la mecánica cuántica y, así mismo, dada la importancia que tiene esta en la visión del mundo, surge la necesidad de plantear una propuesta de enseñanza que permita una mejor comprensión de los diferentes temas que se abordan en mecánica cuántica. En este sentido, se proponen actividades experimentales que permiten a los estudiantes reflexionar en torno al fenómeno mismo y a la vez les proporcione un conocimiento con sentido y significado. 
La actividad experimental planteada es entendida como la estrategia que le permite al estudiante desarrollar habilidades y maneras de proceder para el análisis del fenómeno, permitiéndole construir y reconstruir argumentos para dar cuenta de este. En este sentido, se espera que el estudiante logre desarrollar una explicación en torno al fenómeno, ya que, en general, se presentan dificultades alrededor de los conceptos debido a que los fenómenos cuánticos no son directamente evidentes y, en muchas ocasiones, son contrarios a la intuición de los estudiantes. Bajo este contexto, la actividad experimental es mirada como el vínculo que le permite al estudiante construir explicaciones en torno a los efectos observados.

\section{Perspectiva fenoménica}

El creciente avance que ha tenido la física en los últimos años y la demanda que es exigida para la enseñanza de nuevos temas plantea una serie de interrogantes: 2 Qué papel desempeña la actividad experimental y la formalización para la comprensión de los fenómenos a nivel atómico? ¿̇Cómo se puede promover un pensamiento científico en los estudiantes desde la física que se enseña?

Considerando las diferentes dificultades que los estudiantes tienen en el aprendizaje de la mecánica cuántica y, así mismo, dada la importancia que tiene esta en la visión del mundo (Müller et al., 2002), se hace necesaria una familiarización de esta como lo plantea Dirac (1967):

Las nuevas teorías, si se consideran independientemente de su estructura matemática, están constituidas por conceptos físicos que no pueden expresarse mediante términos o elementos conocidos previamente por el estudiante, y que ni siquiera pueden explicitarse adecuadamente con palabras. Al igual que los conceptos fundamentales (como por ejemplo, proximidad, identidad) que cada uno ha de aprender cuando nace, los nuevos conceptos de la física solo pueden dominarse familiarizándose con sus propiedades y usos. (p. 9).

Por lo tanto, se propone una perspectiva fenoménica en la que la actividad experimental es entendida como el espacio que les permite a los estudiantes realizar una reflexión alrededor del fenómeno para su explicación. A partir de la observación que el estudiante realice, este se va familiarizando cada vez más con el fenómeno, permitiéndole construir y reconstruir conceptos, maneras de pensar y proceder para su explicación, aunque esto depende del grado de madurez y experiencia que el estudiante logre, la explicación que realice es construida a partir de la observación, la cual se va transformando cada vez en la medida en que el estudiante se siente satisfecho con la explicación.

Bajo esta perspectiva, el estudiante se involucra en la formalización realizando una descripción de lo observado y asignando una serie de características y cualidades del fenómeno. La actividad de formalización es entendida como un constructo de palabras que le sirven al estudiante para hablar del fenómeno. En este sentido, se estimula al estudiante a la construcción de fenomenologías que es la estrategia planteada para la enseñanza de la mecánica cuántica y, en particular, sobre la noción de estado, principio de superposición, amplitud de probabilidad y probabilidad.

La actividad experimental resulta útil en este caso ya que por medio de esta el estudiante evidencia una serie de efectos que le permiten hacer una explicación usando un lenguaje que tiene sentido para él. Bajo este contexto, el estudiante construye una experiencia sensible en torno a los efectos observados; efectos que caracteriza alrededor de una cualidad permitiéndole dar una explicación del fenómeno y una formalización de este (Malagón et al., 2013). 


\section{Actividades experimentales}

El propósito de las actividades experimentales es aportar a la enseñanza-aprendizaje de la mecánica cuántica en torno a sus fundamentos básicos. Cada actividad experimental se plantea con el fin de que el estudiante construya una explicación alrededor de los efectos que observa. En este sentido, los aspectos del fenómeno quedan establecidos dada la caracterización que hace el estudiante para su comprensión, además, en cada actividad se enfatiza el abandono del proceder clásico de conocer, persuadiendo al estudiante por un conocer cuántico que le permita construir la explicación.

\section{Actividad experimental I}

En la primera actividad se hace uso de una fuente de luz visible, un colimador y un prisma. La disposición de los elementos se muestra en la figura 1.

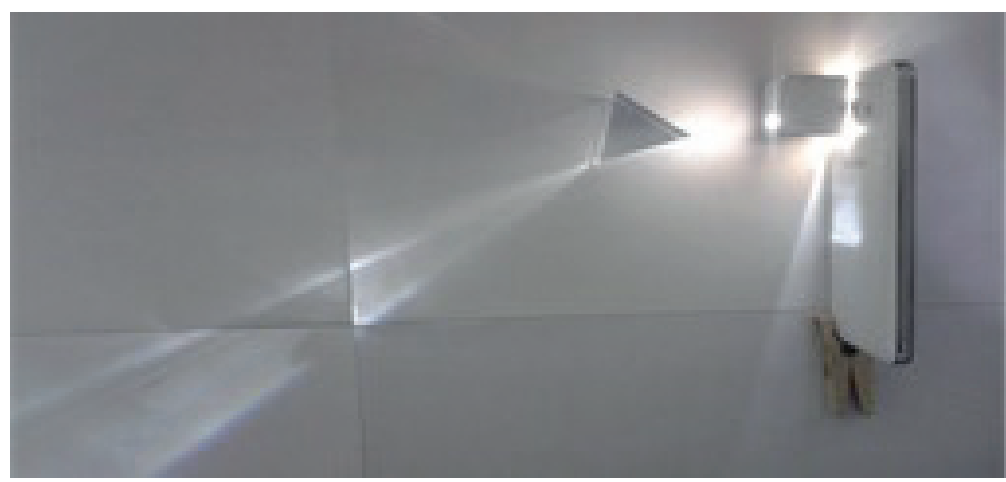

Figura 1. Disposición de la linterna, colimador y prisma

Fuente: elaboración propia.

El haz de luz visible es colimado por una rendija y enfocado hacia el prisma. El estudiante es consciente de la descomposición de la luz por el prisma caracterizándola como una superposición de haces de diferentes colores, es decir, a partir de la actividad se busca que el estudiante construya una experiencia sensible en torno al efecto observado, el espectro de la luz visible.

No obstante, considerando la luz visible como un haz de fotones, y además, suponiendo un solo fotón atravesando el prisma, se puede destacar en este una cualidad: el color, el cual caracteriza a cada fotón del espectro. La cualidad de color permite describir el fenómeno en torno al concepto de superposición de fotones de diferentes colores como se esquematiza en la figura 2. 

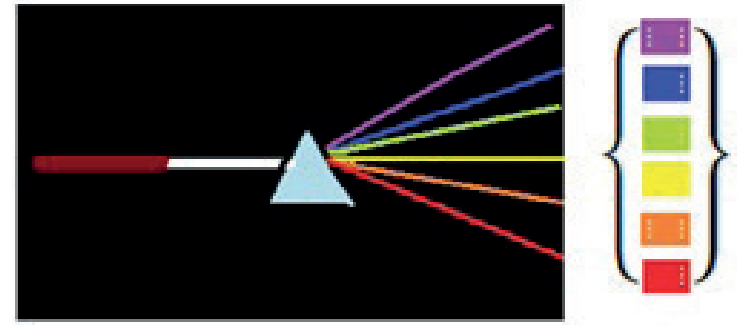

Figura 2. Representación de un fotón atravesando el prisma

Fuente: elaboración propia

El análisis del efecto y el comportamiento del fotón blanco permite hacer una formalización en torno a lo observado, es decir, a partir del esquema de la figura 2 se puede construir la siguiente representación: fotón morado $|M\rangle$; fotón azul $\left|A_{z}\right\rangle$; fotón verde $|V\rangle$; fotón amarillo $\mid A_{m}>$; fotón naranja $\mid N>$; fotón rojo $\mid R>$. A partir de esta representación, el principio de superposición surge naturalmente como la manera de formalizar la relación entre los diferentes fotones, es decir, el estado del fotón blanco $\mid F_{B}$ > puede ser escrito como una combinación lineal del conjunto de fotones $|M>;| A_{z}>; \mid V>$; $\left|A_{m}>;\right| N>; \mid R>$,

$\mid F_{B}>=\frac{1}{\sqrt{6}}\left(|M>+| A_{z}>+|V>+| A_{m}>+|N>+| R>\right)$.

El estado del fotón blanco $\mid \mathrm{L}_{B}>$ no es un estado bien definido sino que, por el contrario, se considera un estado de superposición, un estado constituido por estados bien definidos. El principio de superposición es la adopción de una formalización que se hace para dar cuenta de la organización de la variedad de fotones que se observa. Ahora, żqué tiene de cuántica la actividad experimental? ¿̇Dónde está lo cuántico?

Cuando se considera la luz visible como un haz de fotones, y el fotón blanco se formaliza como la suma de fotones de diferentes colores, es decir, la suma de una variedad de estados de momentum bien definido, es fácil entender que el momentum de cada uno de los fotones solo puede tomar valores discretos en lugar de tomar cualquier valor continuo. En este sentido, lo cuántico está en la variedad de estados de momento bien definido de los fotones, es decir, el observable de momentum solo puede tomar cierto valor discreto para satisfacer todas las relaciones entre observables.

\section{Actividad experimental II}

Esta actividad experimental es igual a la primera, salvo que esta vez se hace uso de dos prismas y dos colimadores. La disposición de los elementos se muestra en la figura 3 .

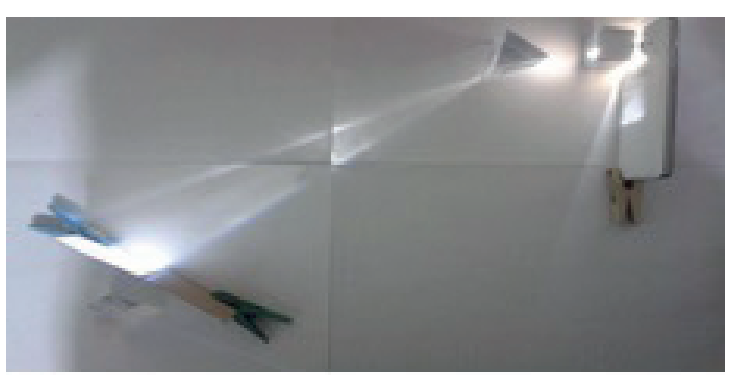

Figura 3. Disposición de la linterna, los colimadores y los prismas

Fuente: elaboración propia.

Dado que en la primera actividad se caracteriza el fotón a partir de la cualidad de color, esta permite introducir la noción de estado definiéndolo como las diferentes formas de estar el fotón en relación con la cualidad. La noción de estado se puede evidenciar colocando placas para bloquear los diferentes haces de fotones y permitiendo el paso de solo uno de ellos, por ejemplo, el fotón azul. Seguidamente, el fotón azul es colimado por una rendija y enfocado hacia el segundo prisma, observando que el fotón azul cuando atraviesa el segundo prisma no se descompone en otros fotones como sí lo hace el fotón blanco. Esto evidencia que cada fotón que compone el fotón blanco puede ser considerado un estado bien definido como se muestra en el esquema de la figura 4. 

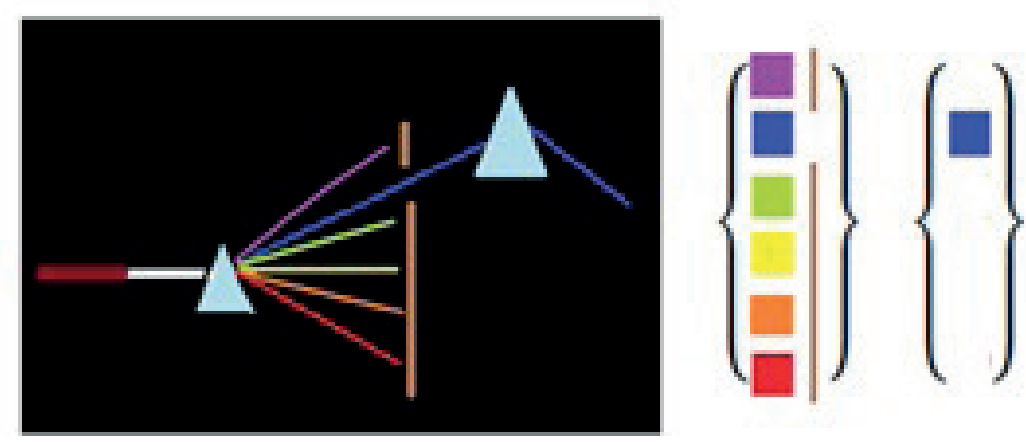

Figura 4. Representación del estado bien definido del fotón azul

Fuente: elaboración propia.

La noción de estado es la estrategia que se utiliza para dar cuenta de las diferentes formas de estar de la luz en relación con la cualidad sin que el haz de luz pierda su identidad. Por lo tanto, la conceptualización sobre la noción de estado y el principio de superposición son consecuencia de la estrategia utilizada para dar explicación del fenómeno haciendo uso de la cualidad específica.

Las actividades I y II permiten evidenciar la formalización de la relación entre los diferentes estados del fotón en cuanto a una cualidad. Por lo tanto, se establece que cada fotón, $|M>,| A_{z}>,|V\rangle,\left|A_{m}>,\right| N>y \mid R>$, se considera un estado bien definido. Además, se puede afirmar que el fotón antes de entrar en el primer prisma se compone de una variedad de estados de momentum cada uno con un momentum bien definido, comprobando, además, que cada fotón es un estado bien definido cuando se hace pasar por un segundo prisma (Feynman, 1964).

Se puede proponer un símbolo para evidenciar los estados bien definidos de la luz blanca logrando que todos los razonamientos sean más simples como se muestra en el esquema de la figura 5.
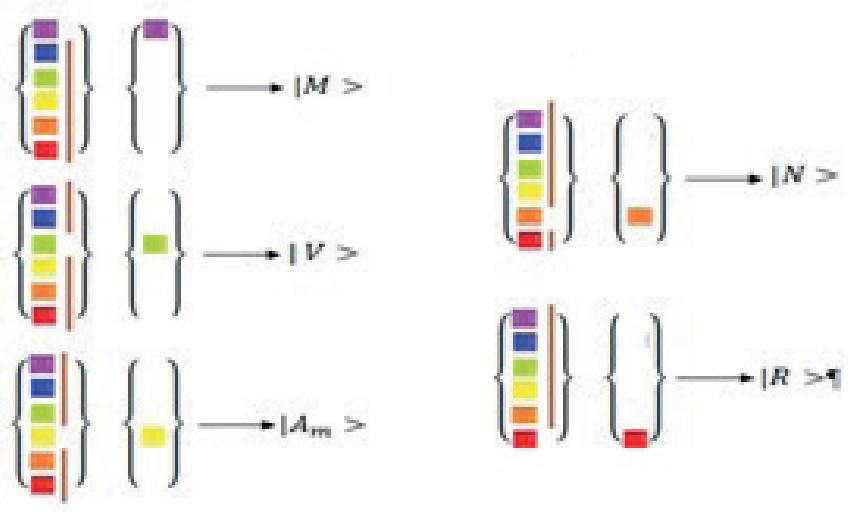

Figura 5. Formalización de los estados, colores, bien definidos

Fuente: elaboración propia. 
Finalmente, la formalización del fenómeno es entendida como aquella en la que el estudiante construye una serie de palabras para referirse a él, caracterizando su comportamiento gracias a los efectos observados. Además, en su caracterización se ve obligado a realizar una clasificación de las cualidades más relevantes con las que puede describir el sistema, que para este caso es el color.

\section{Actividad experimental III}

En la siguiente actividad se hace uso de tres polarizadores y una fuente de luz. La disposición de cada uno de los elementos se muestra en la figura 6.

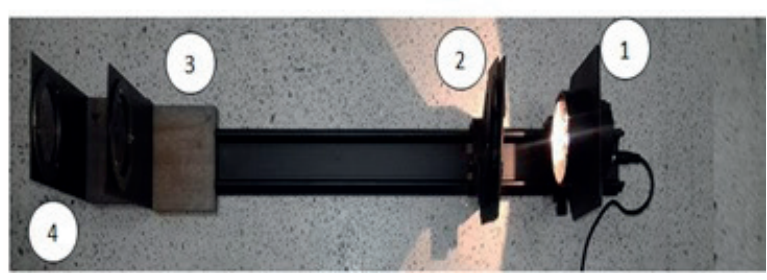

Figura 6. Disposición de la fuente (1), los polarizadores (2), (3) y el polarizador analizador (4) Fuente: elaboración propia.

A partir de esta actividad se busca que el estudiante construya una experiencia sensible a partir de los efectos observados; efectos que el estudiante debe caracterizar en torno a la cualidad de polarización de la luz. El estado de polarización del fotón se puede establecer manteniendo el primer polaroide fijo (2) con respecto a la fuente de luz (1) y rotando a la vez el segundo polarizador (3) con respecto al primero (2). El estudiante percibe un efecto de atenuación en la intensidad de la luz a medida que rota el segundo polarizador, siendo consciente de que al estar el segundo polarizador rotado $90^{\circ}$, con respecto al primero, no observa luz (4), como se muestra en la figura 7.

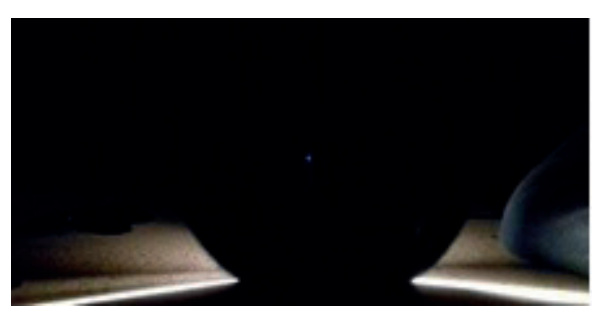

Figura 7. Efecto de atenuación total en la intensidad de la luz cuando el segundo polarizador (3) se encuentra rotado $90^{\circ} \mathrm{con}$ respecto al primero (2)

Fuente: elaboración propia

Tomando los dos estados, $|x>y| y>$, como usualmente se designan, estados de polarización bien definidos, el estudiante puede comprobar el estado de los fotones simplemente observando si sale o no luz a través del segundo polarizador. Por lo tanto, el estado de polarización $|y\rangle$ se obtiene como resultado de tener los dos ejes de los polarizadores orientados en la misma dirección (y) observando luz a través del segundo polarizador, como se muestra en la figura 8.

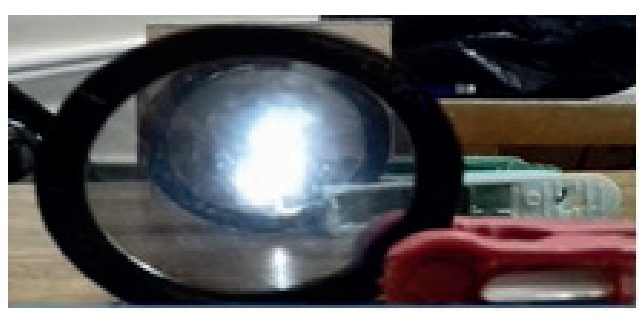

Figura 8. Estado de polarización del fotón

Fuente: elaboración propia.

De igual forma, se puede realizar el mismo análisis para obtener el estado de polarización $\mid x>$ cuando los ejes de los dos polarizadores se encuentran orientados en la dirección $(x)$. Las actividades experimentales anteriores se pueden esquematizar como se muestra en la figura 9. 

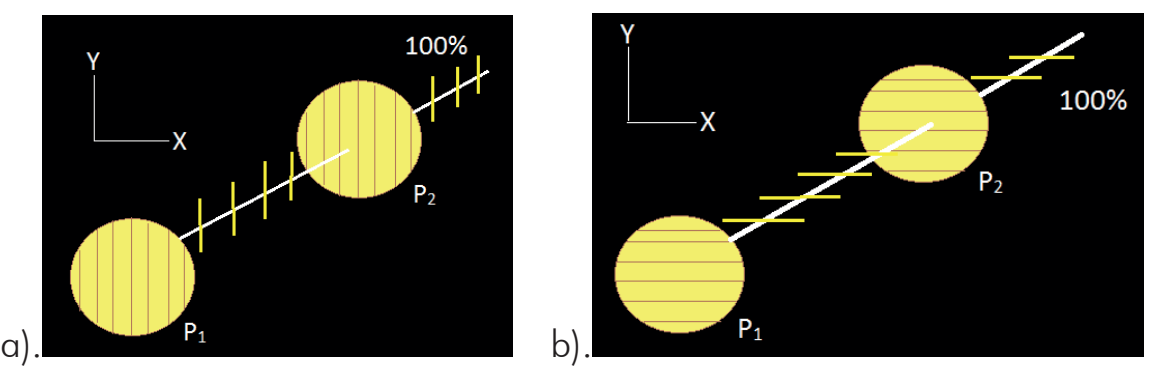

Figura 9. Dos estados de polarización: a). estado de polarización $(y\rangle$, b). estado de polarización $|x\rangle$

Fuente: elaboración propia.

Para el caso en el que se percibe una atenuación total en la intensidad de la luz a través del segundo polarizador, se esquematiza como se muestra en la figura 10. Lo cual significa que un estado de polarización $|y\rangle$ no es un estado de polarización $|x\rangle$, figura 10a. De igual forma, un estado de polarización $|x\rangle$ no es un estado de polarización $\mid y>$, figura $10 b$.

a).

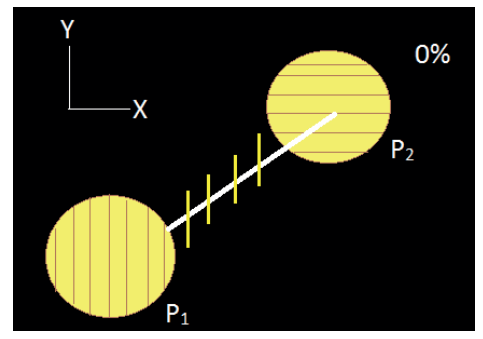

b)

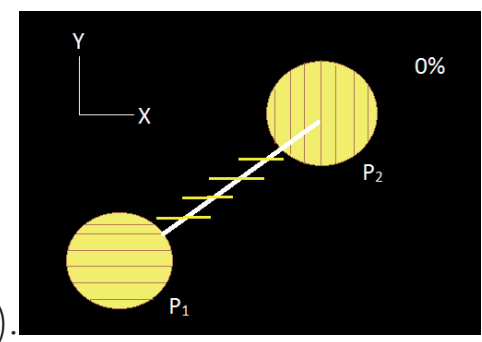

Figura 10. Dos polarizadores: a. el primero $\mathrm{P}_{1}$ con eje de polarización en la dirección (y) y el analizador $\mathrm{P}_{2}$ con su eje de polarización en la dirección $(x)$. b. el primero $\mathrm{P}_{1}$ con eje de polarización en la dirección $(x)$ y el analizador $\mathrm{P}_{2}$ con su eje de polarización en la dirección (y)

Fuente: elaboración propia

A partir de las actividades anteriores se puede hacer una formalización sobre los estados de polarización del fotón. Cuando el eje de polarización se encuentra orientado en la dirección $(y)$ se representa por el símbolo: $\left\{\begin{array}{l}x \mid y \\ y\end{array}\right\}$. De la misma forma, cuando el eje de polarización se encuentra orientado en la dirección $(x)$ se representa por el símbolo: $\left\{\begin{array}{c}x \\ y \mid\}\end{array}\right]$. Los anteriores símbolos muestran de manera abreviada el efecto de los polarizádores cuando el haz de fotones los atraviesa, identificando cada polarizador con una letra debajo, además, las barras verticales simbolizan el bloqueo de una de las orientaciones del polarizador como se muestra en la figura 11.

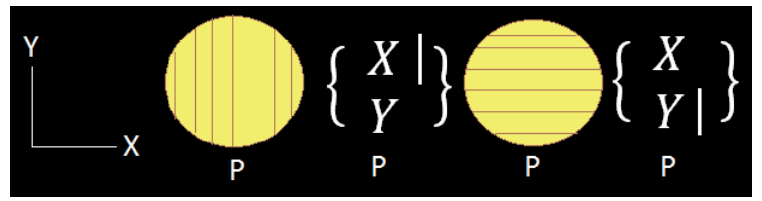

Figura 11. Representación simbólica de los polarizadores

Fuente: elaboración propia. 
Las actividades experimentales pueden ser

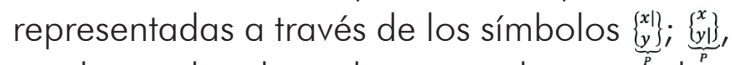
con los cuales el estudiante puede comprobar el estado del fotón de la siguiente forma: cuando los polarizadores que coloca uno junto al otro están orientados en la dirección (y), puede afirmar que el estado del fotón será:

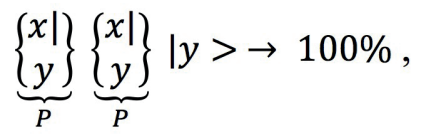

el estado de polarización $\mid y>$ como se muestra en la figura 9a. De igual forma, cuando los polarizadores están orientados en la dirección $(x)$, puede afirmar que el estado del fotón será:

$$
\underbrace{\left\{\begin{array}{c}
x \\
y \mid
\end{array}\right\}}_{P} \underbrace{\left\{\begin{array}{c}
x \\
y \mid
\end{array}\right\}}_{P} \mid x>\rightarrow 100 \%,
$$

el estado de polarización $|x\rangle$ como se muestra en la figura 9b. Los estados de polarización $\mid y>$ y $\mid x>$ obtenidos de esta forma son considerados estados bien definidos del fotón. Ahora, si los ejes de los polarizadores tienen orientaciones diferentes,

$$
\underbrace{\left\{\begin{array}{c}
x \\
y
\end{array}\right\}}_{P} \underbrace{\left\{\begin{array}{c}
x \\
y \mid
\end{array}\right\}}_{P} \rightarrow 0 \%,
$$

significa que un estado de polarización bien definido $|y\rangle$ no es un estado de polarización bien definido $|x\rangle$ como se muestra en la figura 10.

Lo anterior concuerda con las actividades experimentales y permite además sintetizar los resultados obtenidos de la siguiente forma:

$$
\begin{array}{ccc}
<j \mid i> & \mid x> & \mid y> \\
<x \mid & 1 & 0 \\
<y \mid & 0 & 1
\end{array} \quad<j \mid i>=\left\{\begin{array}{lll}
0 & \text { si } & i \neq j \\
1 & \text { si } & i=j
\end{array} .\right.
$$

Este ordenamiento de números, denominado matriz, resume las actividades experimentales que han sido desarrolladas. Además, muestra la condición de ortonormalidad de los estados de polarización $|x\rangle,|y\rangle$, los cuales pueden ser tomados como el conjunto de estados de base para un espacio de dos dimensiones. La anterior formalización es suficientemente consistente con la observación de los efectos apreciados por los estudiantes.

A partir de los estados de polarización bien definidos del fotón se puede introducir el concepto de amplitud de probabilidad. Si un fotón se encuentra en el estado inicial $|x\rangle$ y se quiere saber cuál es la amplitud de probabilidad de que el fotón quede en el estado final $|x\rangle$, al atravesar el segundo polarizador, se formaliza de la siguiente manera: $\langle x \mid x\rangle=1$. Esta expresión indica que el fotón se encuentra inicialmente en el estado $(\mid x>)$ y pasa al estado final $(<x \mid)$. De igual forma, la amplitud de probabilidad para que un fotón estando en el estado inicial $|y\rangle$ quede en el estado final $|y\rangle$ se puede escribir como: $<y \mid y>=1$. Además, la amplitud de probabilidad para que un fotón estando en el estado inicial $\mid y>$ quede en el estado final $|x\rangle$ se puede escribir como: $\langle x \mid y\rangle=0$. Finalmente, la amplitud de probabilidad para que un fotón estando en el estado inicial $\mid x>$ quede en el estado final $\mid y>$ se puede escribir como: $\langle y \mid x\rangle=0$. Las anteriores ecuaciones se pueden representar haciendo uso de los siguientes arreglos:

$$
\begin{aligned}
& \underbrace{\left\{\begin{array}{l}
x \\
y \mid
\end{array}\right.}_{P} \underbrace{\left\{\begin{array}{l}
x \\
y \mid
\end{array}\right\}}_{P}<x \mid x>=1 ;\{\underbrace{\left\{\begin{array}{l}
x \mid \\
y
\end{array}\right\}}_{P} \underbrace{\left\{\begin{array}{l}
x \mid \\
y
\end{array}\right\}}_{P}<y \mid y>=1 .
\end{aligned}
$$

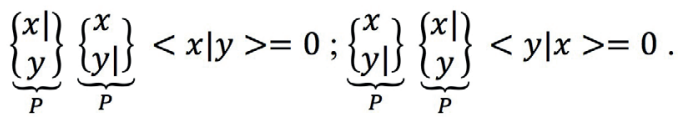

En cada una de las situaciones anteriores se puede establecer la probabilidad de que el fotón estando en un estado inicial termine en un determinado estado final:

$$
\begin{aligned}
& \left.P_{1}=|<y| y>\left.\right|^{2}=\mid \begin{array}{ll}
0 & 1
\end{array}\right)\left.\left(\begin{array}{l}
0 \\
1
\end{array}\right)\right|^{2}=|1|^{2}=1, \\
& \left.P_{2}=|<x| y>\left.\right|^{2}=\mid \begin{array}{ll}
1 & 0
\end{array}\right)\left.\left(\begin{array}{l}
0 \\
1
\end{array}\right)\right|^{2}=|0|^{2}=0, \\
& \left.P_{3}=|<y| x>\left.\right|^{2}=\mid \begin{array}{ll}
0 & 1
\end{array}\right)\left.\left(\begin{array}{l}
1 \\
0
\end{array}\right)\right|^{2}=|0|^{2}=0, \\
& \left.P_{4}=|<x| x>\left.\right|^{2}=\mid \begin{array}{ll}
1 & 0
\end{array}\right)\left.\left(\begin{array}{l}
1 \\
0
\end{array}\right)\right|^{2}=|1|^{2}=1,
\end{aligned}
$$

donde se ha utilizado el siguiente hecho,

$$
|x\rangle=|x\rangle+0|y\rangle=:\left(\begin{array}{l}
1 \\
0
\end{array}\right) ;|y\rangle=0|x\rangle+|y\rangle=:\left(\begin{array}{l}
0 \\
1
\end{array}\right) \text {. }
$$


Los resultados ilustran uno de los principios básicos de la mecánica cuántica: un estado arbitrario del sistema puede ser escrito como una combinación lineal del conjunto de estados base $|x\rangle, \mathrm{y}|y\rangle$,

$$
\mid \beta>=\frac{1}{\sqrt{2}}(|x>+| y>) .
$$

El estado $\mid \beta>$ no es un estado bien definido sino por el contrario se considera un estado de superposición, lo cual sugiere que el análisis anterior pueda ser generalizado cuando uno de los polarizadores tenga una orientación arbitraria con respecto al primero. Finalmente, el anterior análisis le permite al estudiante una clarificación de los conceptos de estado, principio de superposición, amplitud de probabilidad y probabilidad que usualmente se abordan en la enseñanza de la mecánica cuántica y que en muchas ocasiones no tienen sentido ni significado para el estudiante. El siguiente cuadro muestra los resultados obtenidos a partir de las actividades experimentales planteadas.

Tabla 1. Principios básicos de la mecánica cuántica

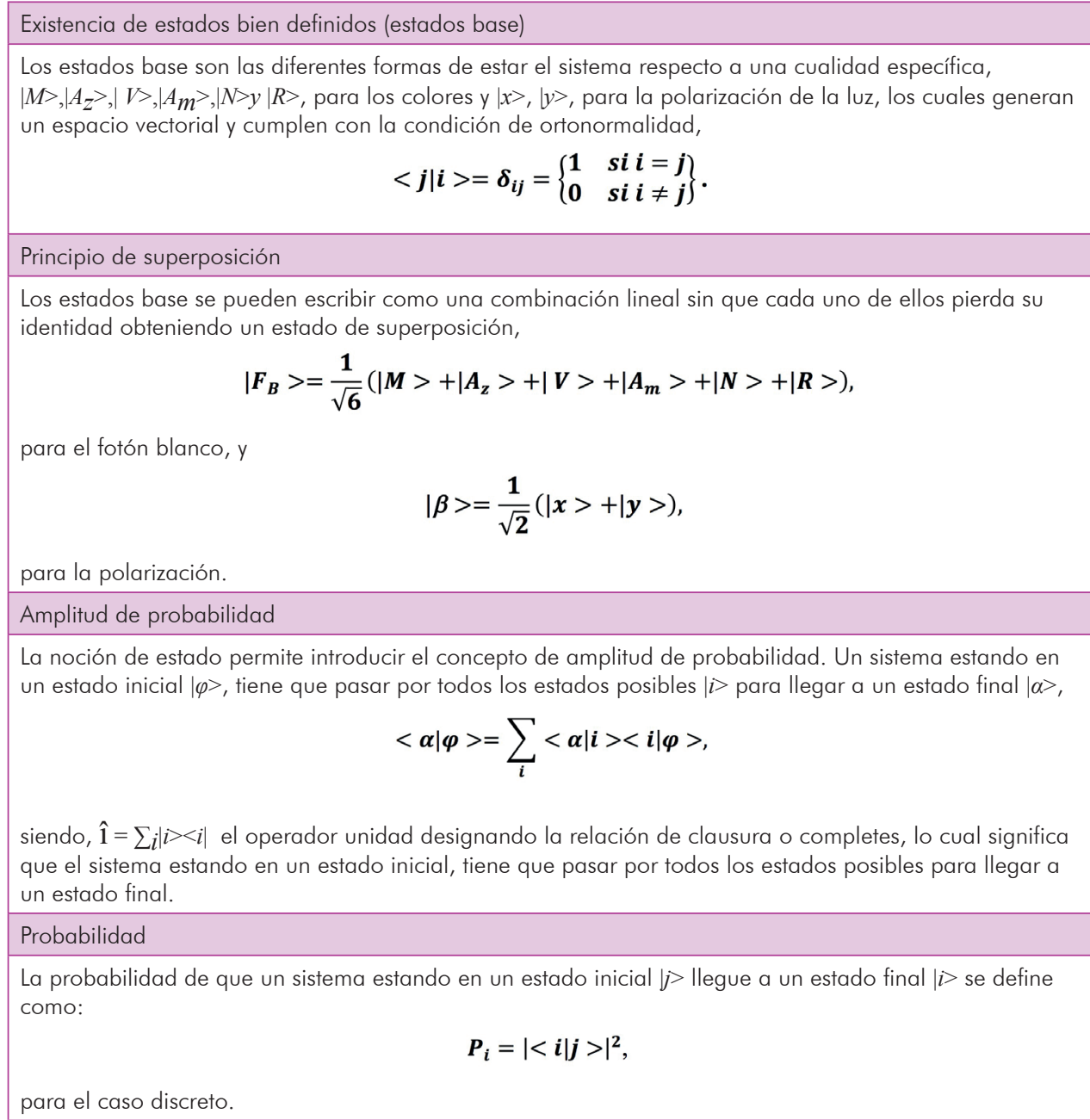

Fuente: elaboración propia 
Finalmente, la anterior formalización hace evidente la relación de constitución con las actividades experimentales planteadas y la metodología propuesta permite hacer una mejor comprensión y explicación de los efectos observados. Por lo tanto, se espera que los estudiantes adquieran un conocimiento con sentido y significado bajo la perspectiva planteada.

\section{Conclusiones}

Los fenómenos cuánticos no son directamente perceptibles y, por lo tanto, son contrarios a la intuición que deriva de la percepción de los estudiantes, esto, por un lado, dificulta la enseñanza de la mecánica cuántica y, por otro, al estudiante le resulta difícil formarse una imagen mental sobre el objeto de estudio.

Se propende estimular en los estudiantes una "intuición cuántica" a partir de las actividades experimentales planteadas, además estas dan la posibilidad de desarrollar una práctica de enseñanza diferente, novedosa y didáctica que permite una mayor clarificación y familiarización sobre la notación y conceptos empleados en mecánica cuántica.

La propuesta se dirige a profesores y estudiantes de secundaria, así como a profesores en formación con el fin de lograr un cambio de paradigma en la enseñanza de física. Además, se busca aportar elementos que permitan una transformación en las prácticas de enseñanza.

Desde esta perspectiva, la formación docente se enmarca dentro de un proceso que promueve un cambio en la formación inicial y permanente de los docentes para la enseñanza de la física y, en particular, de la mecánica cuántica.

Esta forma de plantear la enseñanza de la mecánica cuántica supera la dicotomía entre experimento y teoría ya que desde esta perspectiva el experimento es considerado parte de la construcción de explicaciones en torno al fenómeno.
Este análisis revela cómo el esquema de la mecánica cuántica se convierte en un paradigma para la construcción de explicaciones en torno de los fenómenos a nivel atómico dando la oportunidad de generar una forma alternativa, didáctica y robusta para abordarla con el fin de facilitar su enseñanza-aprendizaje en torno a los principios básicos en los que se fundamenta. Además, este enfoque permite familiarizar a los estudiantes tempranamente con la cosmovisión que ofrece la mecánica cuántica.

\section{Agradecimientos}

Este escrito ha sido realizado con el apoyo de la Universidad Pedagógica Nacional mediante el proyecto DFI-445-17 del Centro de Investigaciones de la Universidad Pedagógica (CIUP).

\section{Referencias}

Beltrán, C. y Mosquera, C. (2016). Algunas reflexiones en torno a las implicaciones de la $\mathrm{NdC}$ en educación en ciencias: el caso de la enseñanza de la mecánica cuántica. Tecné, Episteme y Didaxis, número extraordinario. ISSN impreso: 0121-3814, ISSN web: 2323-0126. Memorias, Séptimo Congreso Internacional sobre Formación de Profesores de Ciencias. 12 al 14 de octubre del 2016, Bogotá.

Dirac, P. A. M. (1967). Principios de mecánica cuántica. Barcelona: Ariel.

Fanaro, M. (2009). La enseñanza de la mecánica cuántica en la escuela media. (Tesis Doctoral). Universidad de Burgos, Burgos.

Ferreyra, A. y González, E. (2000). Reflexiones sobre la enseñanza de la física universitaria. Enseñanza de las Ciencias, 18(2), 189-199.

Gill, D. (1983). Tres paradigmas básicos en la enseñanza de las ciencias. Enseñanza de las Ciencias, 1, 26-33.

Greca, I. y Herscovitz, E. (2002). Construyendo significados en mecánica cuántica: fundamentación y resultados de una propuesta innovadora para su introducción en el nivel universitario. Enseñanza de las Ciencias, 20(2), 327-338. 
Hodson, D. (1994). Hacia un enfoque más crítico del trabajo de laboratorio, Investigación y experiencias didácticas. Enseñanza de las Ciencias, 12(3), 299-313.

Joshua, S. y Dupin, J. (2005). Introducción a la didáctica de las ciencias y la matemática. Buenos Aires: Colihue.

Malagón, F., Sandoval, S. y Ayala, M. (2013). La actividad experimental: construcción de fenomenologías y procesos de formalización. Praxis Filosófica, 36, e 1 19-138.

Mengual, C. (2005). La imagen compleja: la fenomenología de las imágenes en la era de la cultura visual. Barcelona: Universidad Autónoma de Barcelona.

Moreira, M. (2000). Física contemporánea en la escuela secundaria: una experiencia en el aula involucrando formación de profesores. Enseñanza de las Ciencias, 18(3), 391-404.

Moreira, M. (2004). Sobre cambio conceptual, obstáculos representacionales, modelos mentales, esquemas de asimilación y campos conceptuales. Porto Alegre: UFRGS.

Müller, R. y Wiesner, H. (2002). Teaching quantum mechanics on an introductory level. American Journal of Physics, 70(3), 200-209.

Otero, M., Fanaro, M. y Arlego, M. (2009). Investigación y desarrollo de propuestas didácticas para la enseñanza de la física en la escuela secundaria: Nociones cuánticas. Revista Electrónica de Investigación en Ciencias, 4, 58-74.

Schmid, B. (1984). An up-to-date approach to physics. American Journal of Physics, 52(9), 794-799.

Solbes, J. y Sinarcas, V. (2010). Una propuesta para la enseñanza aprendizaje de la física cuántica basada en la investigación en didáctica de las ciencias. Revista de Enseñanza de la Física, 23(1 y 2), 57-84.

Solbes, J., Bernabeu, J., Navarro, J. y Vento, V. (1988). Dificultades en la enseñanza/ aprendizaje de la física cuántica. Revista Española de Física, 2, 22-27.

\section{Para citar este artículo}

Rozo, M., Walteros, A. y Cortés C. (2019). La actividad experimental como una parte fundamental para la enseñanza de la física moderna: el caso de la mecánica cuántica. Tecné, Episteme y Didaxis: ted, 45, 191-206. 